\title{
Numerical modelling of architectonic structures' thermal response. Laboratory and in-situ data analysis
}

\author{
by R. Di Maio*, C. Mancini*, C. Meola** and E. Piegari* \\ *Department of Earth Sciences, University of Naples “Federico II”, Naples, Italy, rosa.dimaio@unina.it, \\ ceci.mnc@gmail.com, esterpiegari@gmail.com \\ **Department of Aerospace Engineering, University of Naples “Federico II”, Naples, Italy, carmeola@unina.it
}

\begin{abstract}
Numerical codes were developed to model the heat transfer in inhomogeneous media, both in $1 \mathrm{D}$ and $2 \mathrm{D}$ cases, by solving the forward thermal problem through the Finite Difference method. Such codes were first validated through Pulse Thermography measurements on a specimen simulating a damaged wall structure. Then, the codes were applied for quantitative analysis of experimental data acquired in the Marcus Fabius Rufus' House (Pompeii, Italy). In particular, modelling of temperature transients allowed to define both nature and depth of the thermal anomaly sources, which provides important contributions to possible future restoration.
\end{abstract}

\section{Introduction}

Pulse-thermography (PT) is a contactless active method for fast and efficient non-destructive testing of materials or structures. Measurements with PT are performed by thermally stimulating, by a heating pulse, the surface of the medium under investigation, and monitoring, by an infrared camera, the temperature variations on its surface during both heating and cooling transient phases [1-3]. The heat propagation depends on material properties, like thermal conductivity, heat capacity and density. The presence of inhomogeneities (e.g. voids, delaminations, moisture) characterized by different thermal properties with respect to the sound material, affects the surface temperature map permitting their detection. An accurate interpretation of the anomaly sources in terms of size, position and physical properties, is possible only by a quantitative interpretation of the acquired thermal data $[1,4]$.

Nowadays, quantitative analysis for defect characterization is a topic of great interest in non-destructive testing. The most used procedures are based on numerical models that solve the forward thermal problem, i.e. they find the solution of the Fourier differential equation, which describes the heat transfer in a medium. These models aim at assessing physical and geometrical characteristics of eventual anomaly sources by comparing theoretical and experimental temperature transients (inverse problem).

Initially, analytical inversion algorithms were developed to characterize thermal anomalies through transient thermographic imaging under 1D thermal flow conditions $[5,6]$. In this approach an iterative procedure based on the least squares method is used to minimize the deviation between experimental thermal transient data and the corresponding computed analytical transient. Due to the limits of the 1D assumption, numerical Finite Difference models were developed to study two-dimensional thermal flows [6-8]. Concurrently, numerical models based on the Finite Element method were also developed for modelling complex structure specimens. Some authors highlighted the importance of a non-uniform heating, which represents one of the main problems for the comparison of experimental and numerical results, and for identifying the defect characteristics [9]. Others studied extensively initial and boundary conditions to evaluate all necessary input parameters and functions in the case of landmine detection, in which the heating source is the sun and it is important to evaluate the influence of weather and soil conditions $[10,11]$. More recently, a semi-analytic reformulation of the heat transport problem has been proposed for a quantitative detection of defects in test specimens [12]. In this case, the authors capture the boundary layer with an analytically obtained approximation, and compute only the remaining defect numerically in order to increase reliability for quantitative prediction and simultaneously decrease computational costs.

The Finite Element method has also been used to generate synthetic data employed as the input parameters to train neural networks for defect depth estimation in passive and active thermography schemes [13-15].

Alternatively from previous approaches, which search for calibration curves to characterize typical defects in concrete structures, we investigate the possibility of using numerical models based on the Conservative Finite Difference method for a quantitative interpretation of in-situ thermographic data on architectonic structures. Thus, in this work, we show the results of numerical codes derived by the solution of the forward thermal problem to model the heat transfer in inhomogeneous media, both in 1D and 2D cases. First, the codes were validated through laboratory tests on a specimen simulating a wall structure with typical defects that may be encountered in the evaluation of its state of preservation (e.g., voids, breakups, micro-cracks, moisture, etc.). We obtained the temperature transient curves and studied the model response by varying nature, size and depth of defects. Finally, we discuss the results obtained applying the developed codes for modelling in-situ PT data acquired in the Marcus Fabius Rufus' House (Pompeii, Italy).

\section{1D and 2D numerical modeling}

Numerical codes for quantitative interpretation of pulse-thermography data were developed using the Finite Difference (FD) method. The FD method, which is one of the several techniques utilized for obtaining numerical solutions 
to partial differential equations, bases on replacement of the 1st and 2nd partial derivatives with the so called difference equations. The latter involve only the discrete values associated with some points in the domain in which the problem is defined. The discrete approximation results in a set of algebraic equations that are solved for the values of the discrete unknowns [16].

Therefore, we need to firstly specify a grid to conveniently discretize both spatial and time domains (section 2.1) and then to evaluate the approximate heat equation in the grid nodes (section 2.2).

\subsection{Meshing}

The mesh is the grid of points where the solution of the discrete heat equation is calculated in time. Therefore, the interval between consecutive time steps and the local distance between adjacent points of the considered continuous medium have to be defined.

In our case, the temporal steps, $\Delta t$, are constant because the thermal images are acquired at regular time intervals, i.e.:

$$
\Delta t=\frac{t_{f}-t_{0}}{n_{t}-1},
$$

where $t_{0}$ and $t_{f}$ are, respectively, the start and the final time [s] of the cooling phase and $n_{t}$ is the number of time steps.

For defining the spatial sampling, we use two different grids that describe the 1D and 2D case:

- 1D: a point on the surface thermal map is considered and the medium is represented as a line (figure 1).

- 2D: a line on the surface thermal map (e.g. along the $x$ direction) is considered and the medium is represented as a grid, where $z$ is the depth and $x$ is the chosen direction.

To ensure conservation of heat flow between nodal points and to allow a correct numerical solution, we use the Conservative Finite Difference discretization, which allows to model inhomogeneous medium described by heat conservation equation with variable physical properties [17]. The Conservative FD discretization uses a particular grid, named "staggered grid", which includes basic nodes and additional nodes. In basic nodes (orange squares in figure 1), density, specific heat and heat equation are defined, whereas in additional nodes (yellow circles in figure 1), thermal conductivity and heat fluxes are defined.
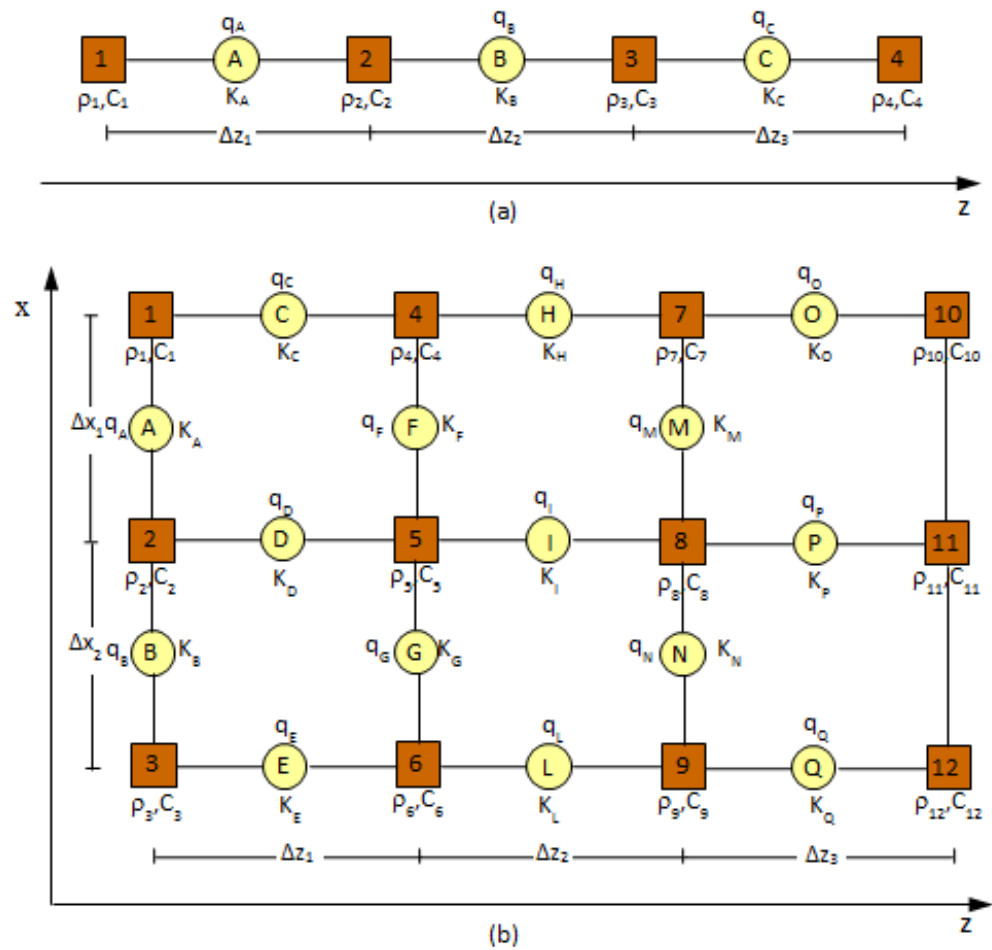

Fig. 1. $1 D$ staggered grid (a) and $2 D$ staggered grid (b). The orange squares are the basic nodes and the yellow circles are the additional nodes

\subsection{Mathematical model}

Let's consider the heat diffusion equation in a non-deforming medium with constant thermal conductivity $(k)$ [17]: 


$$
\rho C \frac{\partial T}{\partial t}=k \nabla^{2} T
$$

where $\rho$ and $C$ are, respectively, the density and the specific heat of the medium.

The implicit 1D Finite Difference discretization in the case of homogenous medium is given by

$$
\frac{T_{i}^{n}}{\Delta t}-\frac{k}{\rho C}\left(\frac{T_{i-1}^{n}-2 \cdot T_{i}^{n}+T_{i+1}^{n}}{\Delta z^{2}}\right)=\frac{T_{i}^{n-1}}{\Delta t},
$$

where $i$ is the spatial node index and $n$ is the time step index.

In the general case of inhomogeneous medium, the spatial steps along the depth direction, $\Delta z_{i}$, are not equal and, as mentioned above, we use the Conservative Finite Difference method, which produces a proper solution of the heat equation.

In the 2D case, the procedure is the same but the discretized equation depends on $\Delta z_{i}$ and $\Delta x_{j}$ (see figure $1 \mathrm{~b}$ ).

Finally, in order to solve the problem, boundary conditions, i.e. the initial temperature values at all the grid nodes have to be fixed. In particular, the initial temperature of the first node is fixed equal to the maximum temperature value of the heating phase, indeed the heating turn-off time coincides with the cooling start time. If the thermal equilibrium is not reached, the last node has a constant temperature for the whole cooling phase, therefore, the initial temperature of this node is set equal to the environmental temperature. Finally, the initial temperatures of the internal nodes are fixed equal to values between the initial temperature of the first node and the last node, these values depend on the materials and/or defects thermal properties.

\section{Laboratory data analysis}

We first validated the developed numerical codes on the thermal response of a specimen (figure 2), which simulates a wall structure with typical defects that may be encountered in the evaluation of its state of conservation (e.g., voids, breakups, micro-cracks, etc.).

The sample of dimension $900 \times 900 \mathrm{~mm}^{2}$ is a two-layer structure consisting of a plaster layer over a support of marble $30 \mathrm{~mm}$ thick. To simulate detachments of the plaster from the support, defects, such as cork disks (orange circles in figure 2a) and air-filled plastic bags (grey circles in figure 2a), were positioned between the support and the plaster. The defects were manufactured of three different diameters $(40,60$ and $100 \mathrm{~mm}$ ) with thickness of approximately 1.5 $\mathrm{mm}$ for cork disks and $2 \mathrm{~mm}$ for plastic bags. In particular, the cork disks simulate break-up of the material by virtue of their natural porous structure. Conversely, the air-filled bags, which consist of two heat welded circles with trapped air, well simulate the presence of voids or micro-cavities.

The specimen is divided in two parts ( $A$ and $B$ ) that include an equal number of defects (see figure 2a). The plaster thickness is $10 \mathrm{~mm}$ on side A, while it is $20 \mathrm{~mm}$ thick on side B.

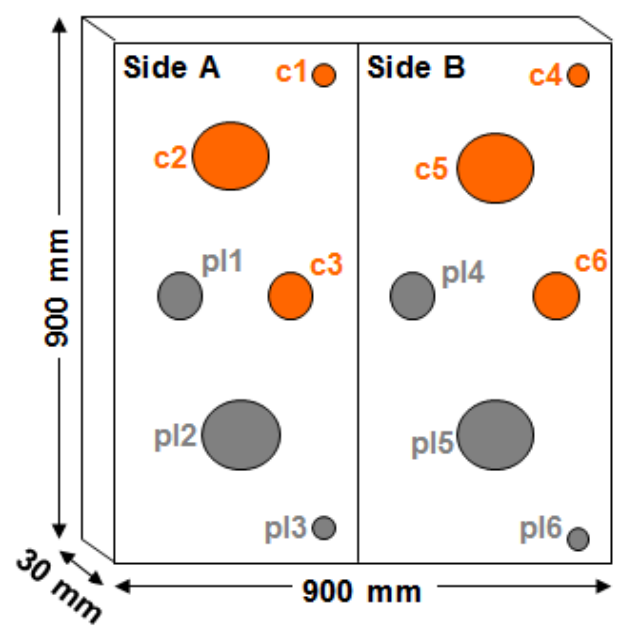

(a)

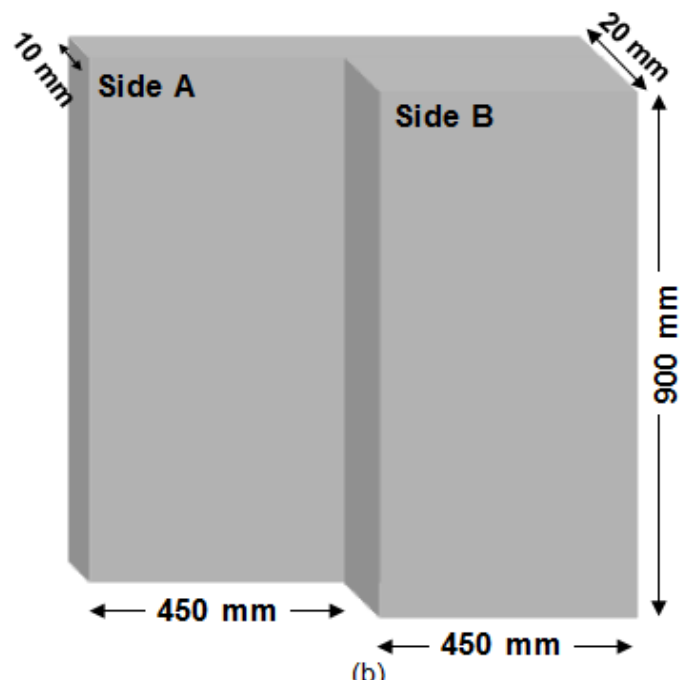

(b)

Fig. 2. Specimen scheme: defects on marble support (a) and plaster layer on the defects (b)

Measurements were performed with pulse-thermography in reflection mode. The upper face (plaster) of the specimen was thermally stimulated by an halogen lamp of $1.5 \mathrm{~kW}$. Sequences of thermal images were acquired with the Flir SC6000 infrared camera at a time interval of $10 \mathrm{~s}$ during both heating (1850 s) and cooling (4200 s) phases. 
Two thermal images taken during the cooling phase are shown in figure 3. As can be seen, $140 \mathrm{~s}$ after starting of the cooling phase (figure 3a), all the defects on the left side, i.e. under the thinner plaster, are well visible, while the defects on the right side, i.e. under the thicker plaster, appear defocused. Conversely, 520 s later (figure 3b), the defects visibility improves on the thicker side and worsens on the thinner one. This feature is justified by the delay with which the heat reaches the top of the defects on the side $B$ and consequently the maximum contrast is shown later than on the side A. In addition, we note that the plastic defects are better distinguishable than the cork ones because the air volume entrapped by cork is smaller than that included in the plastic bags.
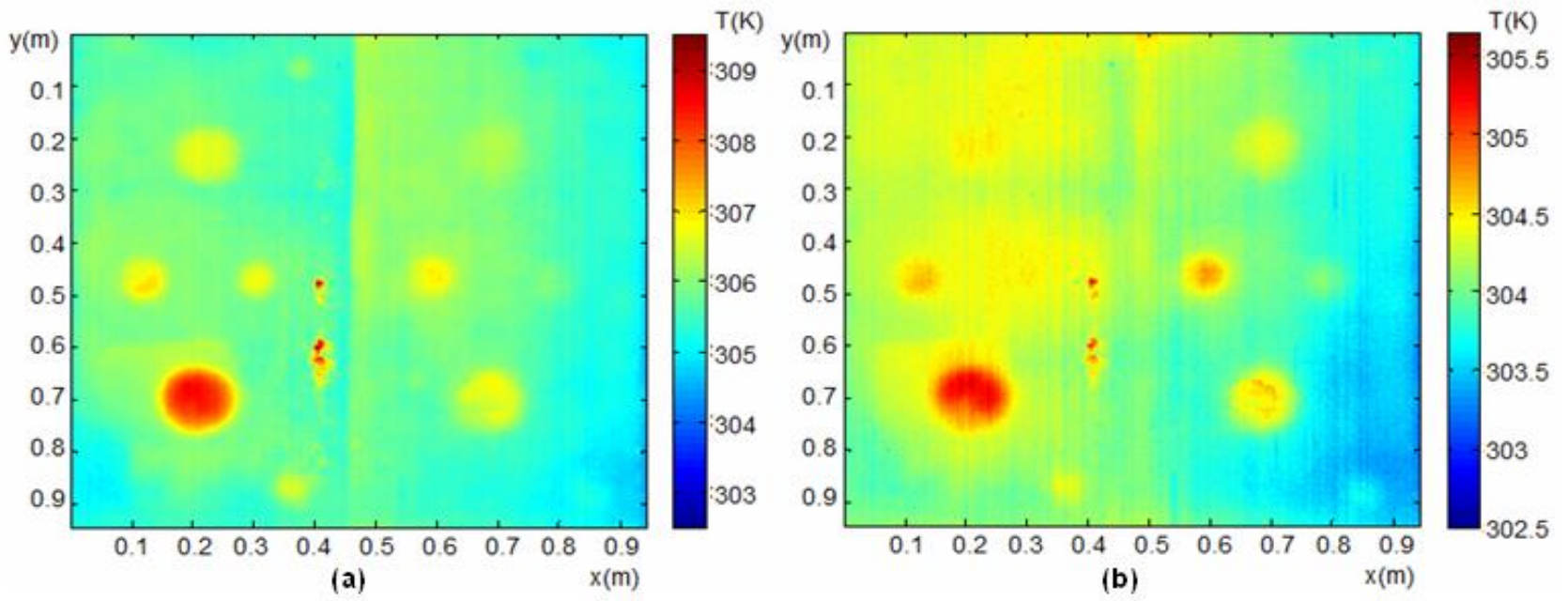

Fig. 3. Thermal image registered at $140 \mathrm{~s}$ (a) and $660 \mathrm{~s}$ (b) after cooling phase started

\subsection{Numerical Simulations}

To validate the developed numerical codes, 1D and 2D simulations were compared with the experimental data obtained from laboratory thermal images acquired during the cooling phase. Explicitly, temperature transients of specific anomalous thermal pixels were compared with the theoretical transient curves coming from the numerical simulations. For the defected zone, we considered the thermal pixels coinciding with the midpoint of the anomaly visible on the thermal map, while for the sound zone (i.e. two-layers made of plaster and marble), the average value amongst different pixels was considered.

The numerical simulations were performed for the whole cooling phase of the experimental test (4200 s) by using a time step, $\Delta t$, of $10 \mathrm{~s}$ and the physical properties (thermal conductivity, density and specific heat) of the specimen materials (table 1). On the other hand, the spatial steps were fixed according to depth and geometry of the defects, and to the thickness of each layer of the specimen.

Table 1. Material physical parameters used for the numerical simulations. In the last column $e_{f}$ is the thermal effusivity

\begin{tabular}{|c|c|c|c|c|}
\hline & $\mathbf{k}[\mathbf{W} /(\mathbf{m} \cdot \mathbf{K})]$ & $\mathbf{\rho}\left[\mathbf{k g} / \mathbf{m}^{\mathbf{3}}\right]$ & $\mathbf{C}[\mathbf{J} /(\mathbf{k g} \cdot \mathbf{K})]$ & $\mathbf{e}_{\mathbf{f}}\left[\mathbf{J} / \mathbf{m}^{2} \mathbf{K s} \mathbf{s}^{\mathbf{1 / 2}}\right]$ \\
\hline Plaster & 0.25 & 1600 & 860 & 586.52 \\
\hline Plastic defect & 0.16 & 1350 & 1480 & 565.4 \\
\hline Cork defect & 0.08 & 480 & 1760 & 259.97 \\
\hline Marble & 2.3 & 2750 & 800 & 2249.44 \\
\hline
\end{tabular}

Finally, we imposed the following boundary conditions (see section 2.2): the temperature of the starting cooling phase (coinciding with the maximum temperature of the heating phase) to the first node. A constant temperature, which is equal to that of the environment, to the last node (this because the marble base of the specimen was not reached by the heat flow during our experiment). Temperatures of the internal nodes were fixed between the initial temperature of first and last nodes; in particular, the internal temperatures were chosen on the basis of the materials thermal properties reported in table 1.

We studied the temperature behaviour of the surface node, $T^{\delta}$, which is the first node of the 1D (or 2D) model. The simulations were performed considering all the defects inserted on both the specimen sides $A$ and $B$ by using models with different numbers of principal and additional nodes. The obtained results show a good agreement between experimental, $T^{e}$, and theoretical, $T^{s}$, transient curves for all the considered anomalous areas. The mean percentage error was evaluated according to the following formula: 


$$
e=\frac{1}{n_{t}} \sum_{i=1}^{n_{t}} \frac{\left|T_{i}^{e}-T_{i}^{s}\right|}{T_{i}^{e}} \cdot 100,
$$

where $n_{t}$ indicates the number of measurements acquired with a time step of $10 \mathrm{~s}$.

As an example, figure $4 \mathrm{a}$ shows the comparison between the experimental temperature decay curve (blue line) for the plastic defect pl1 on side A (see figure 2) and the 1D (red line) and 2D (green line) theoretical curves. For both cases, there is a good agreement, but 1D analysis provides a lower error. Conversely, for the defect pl4 on side B (see figure 2) the lowest error is obtained with the 2D simulation (figure $4 \mathrm{~b}$ ). The modeling of all the defected areas shown in the thermal maps of figure 3 revealed that a 2D modeling is required when the ratio between depth and size of the defect is large enough to appreciate the lateral limits of the defect.
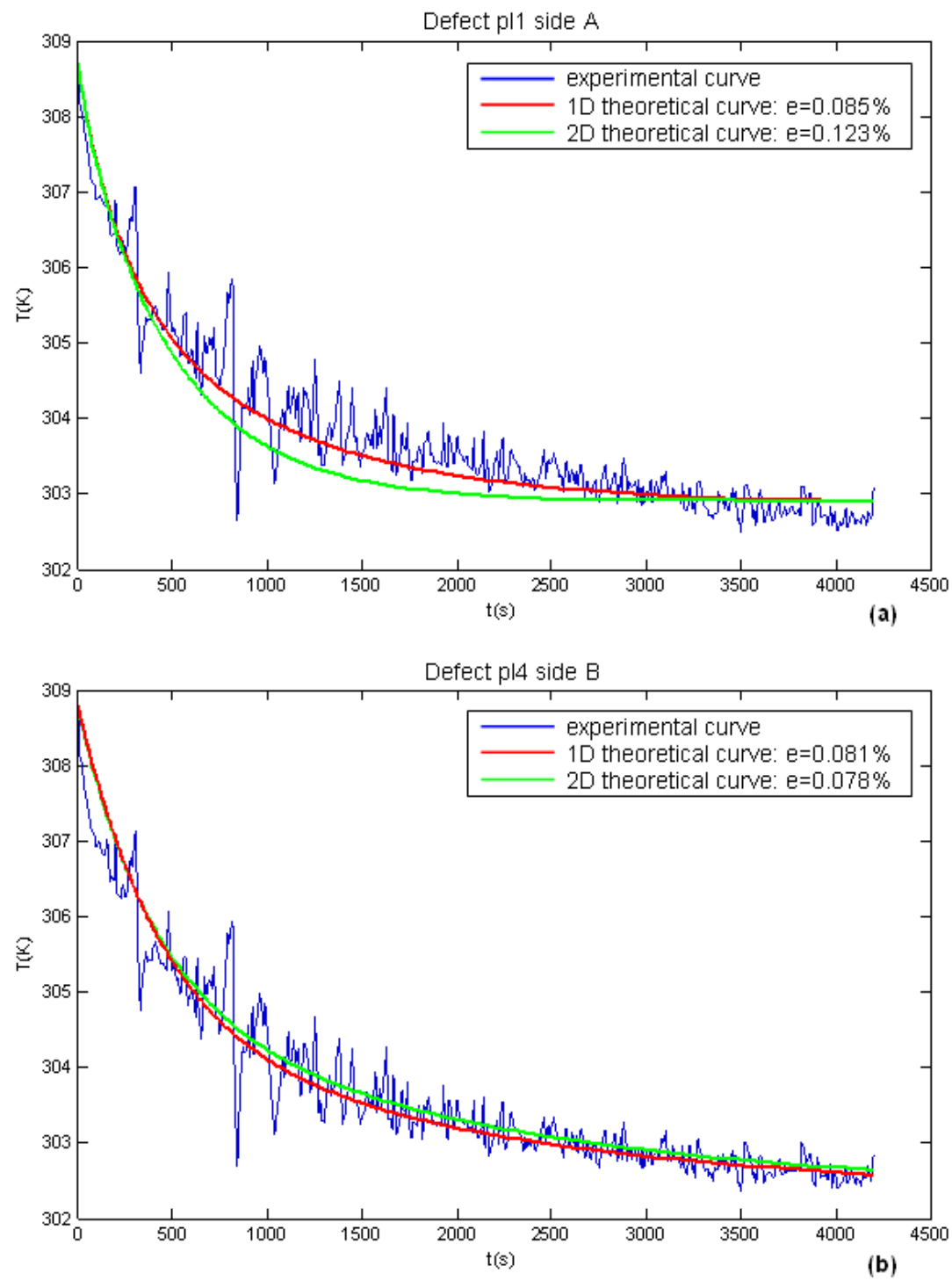

Fig. 4. Comparison between the experimental and theoretical temperature curves corresponding to the plastic defect pl1 on side $A(a)$ and the plastic defect pl4 on side $B(b)$ for the $1 D$ and $2 D$ modeling. The legend also indicates the percentage error (e)

In order to assess the possible limits of the developed codes, iterative cycles were implemented to produce theoretical temperature curves for varying depth and nature of defects. Table 2 shows results for the plastic defect pl4 of figure 2, whose top surface is located at a depth of $1.8 \mathrm{~cm}$ from the specimen surface. As we can see, the nature and the depth of the defect are estimated with a high accuracy level. It is worth to underline that for all the examined defects, such analysis has provided the lowest error in correspondence with the real nature of the defects and an estimation of their depth with an error lower than $5 \%$. 
Table 2. Results of 2D numerical simulations by varying the depth and the nature of the defect pl4 of figure 2

\begin{tabular}{|c|c|c|c|}
\hline Defect depth $\mathbf{( c m )}$ & $\mathbf{e ~ ( \% )}$ & Defect nature & e (\%) \\
\hline $\mathbf{1 . 8 0}$ & $\mathbf{0 . 0 7 8 8}$ & plastic & $\mathbf{0 . 0 7 8 8}$ \\
\hline 1.70 & 0.0828 & air & 0.3722 \\
\hline 1.75 & 0.0801 & water & 0.1924 \\
\hline 1.85 & 0.0787 & lightweight plaster & 0.1294 \\
\hline 1.90 & 0.0800 & dense plaster & 0.0811 \\
\hline 1.95 & 0.0822 & wet plaster & 0.1657 \\
\hline
\end{tabular}

\section{In-situ data analysis}

The numerical codes were applied to pulse-thermography data acquired in-situ on an archaeological building with the aim to provide a quantitative interpretation of anomaly sources very likely correlated to the degradation of wall structures. In particular, the PT measurements were carried out, with the handheld camera T640 (Flir systems), on some inner walls of the Marcus Fabius Rufus' House (figure 5a), located in the archaeological area of Pompeii (Naples, Italy). The House is organized on four levels gradually descending toward the sea and is a typical example of a city villa, equipped with central gardens for each housing level [18]. Doubtless, it represents one of the best examples in the architectonic panorama of Pompeii.

The typical problems of this complex are: water infiltration, degradation of the building materials and inner inhomogeneities [19]. The inspection with infrared thermography was carried out in two rooms of the House, the northeast wall of the room 82 (figure $5 b$ ) and the south-west wall of the room 80 (figure $5 c$ ). The first wall is decorated with dark painting in III Pompeian style and most likely hides an opening, which, in the past, allowed access to the environment 80 . The latter has a rectangular section with a window on the west wall and a floor in tessellatum with white background and a black band.

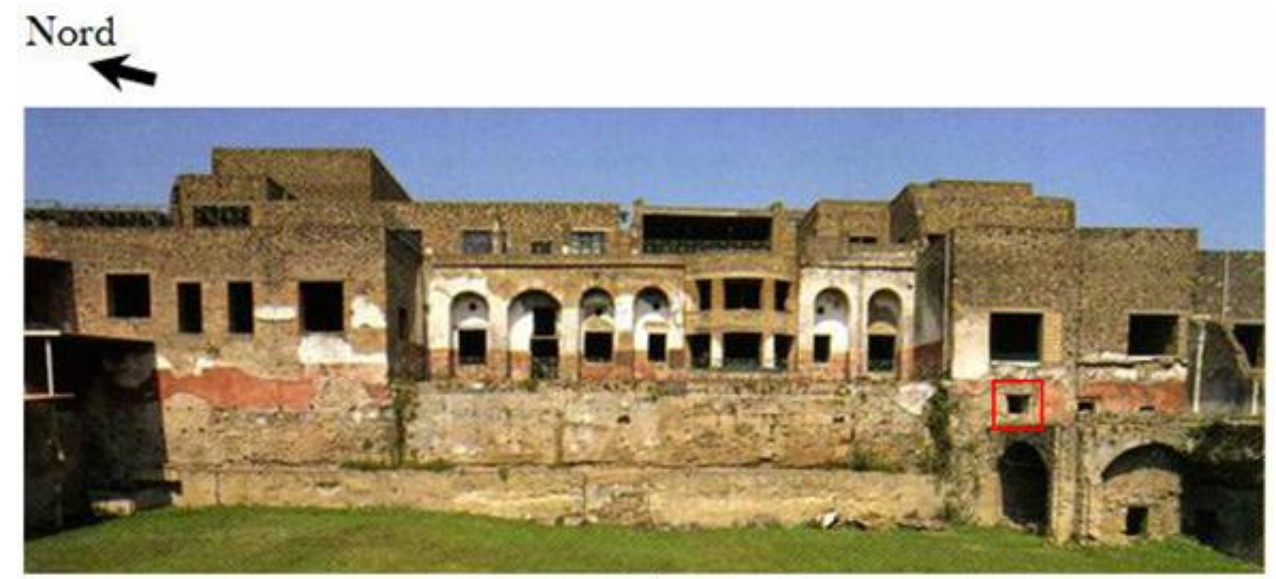

(a)

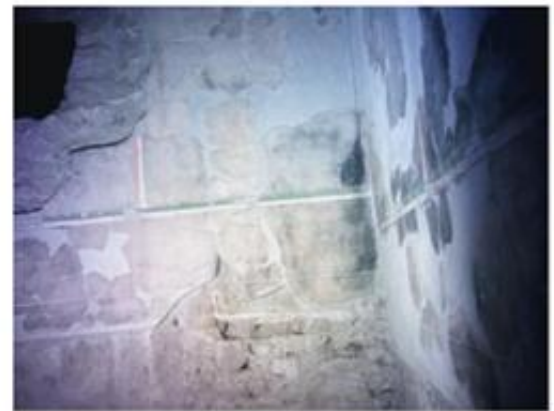

(b)

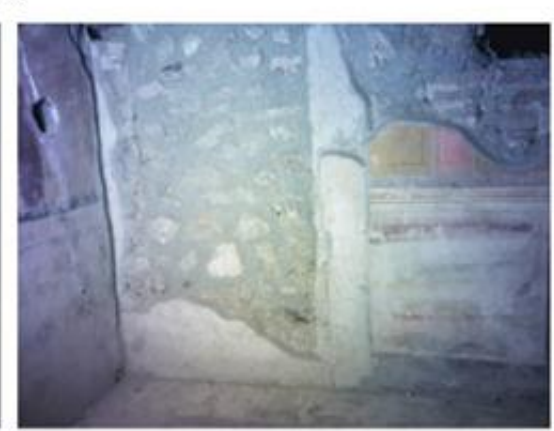

(c)

Fig. 5. (a) Marcus Fabius Rufus' House. The red rectangle indicates the investigated area; (b) north-east wall of the room 82; (c) south-west wall of the room 80.

The PT inspection was carried out in reflection mode with thermal stimulation performed with a halogen lamp $1.5 \mathrm{~kW}$ positioned in front of the selected walls. A sequence of thermal images was acquired during both heating (1200 s) and cooling (1800 s) transient phases at time intervals of $20 \mathrm{~s}$. 
Figure 6 shows two thermal images acquired during the cooling phase on the two investigated walls. The map shown in figure $6 \mathrm{a}$, which refers to the north-east wall of the room 82, exhibits hot anomalies that are due to presence of voids, i.e. to disaggregation of the masonry structure. Conversely, the relatively low temperature anomaly, which is pointed out with an ellipse on the right side of figure $6 \mathrm{a}$, indicates presence of moisture in the disaggregated masonry. Instead, the thermal contrasts that characterize the thermal image, shown in figure $6 \mathrm{~b}$ and which refers to the left side of the south-west wall of the room 80, well describe the contrast between the different building materials, i.e. tuff bricks (yellow areas) and mortar (red areas). Interestingly, no thermal anomalies are evident along the doorjamb (black rectangle in figure $6 \mathrm{~b})$, which, as observed in-situ, is constituted by marble ( $M$ in figure $6 \mathrm{~b})$ with a plaster cover ( $P$ in figure $6 \mathrm{~b}$ ) of about $4 \mathrm{~cm}$ of thickness. Therefore, in order to validate the codes, we have chosen to model the thermal response of the lower sector of the jamb that apparently does not show thermal contrast with respect to the upper part, where the marble was brought to light by the fall of the plaster.
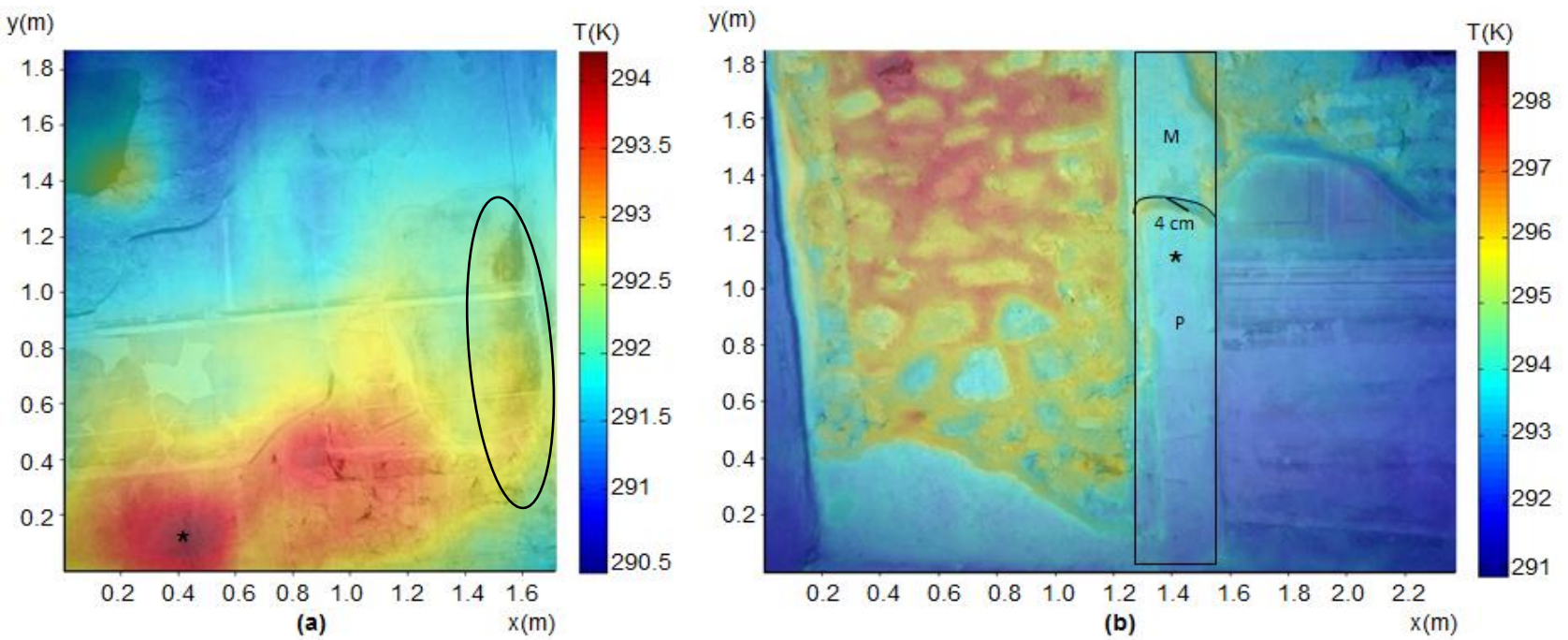

Fig. 6. Thermal images acquired on the north-east wall of the room 82 (a) and on the south-west wall of the room 80 (b) (see figure 5)

\subsection{Numerical Simulations}

The developed numerical codes were applied to model the main thermal anomalies shown in the maps of figure 6. For each defective zone, a model that could justify the observed anomalies was hypothesized, and then the corresponding theoretical thermal response was calculated and compared with the experimental transient. It is worth noting that, as the model was not a priori known, assumptions based on the knowledge of masonry materials typical of Pompeian buildings [20] were made (see table 3).

As examples, we show the results of two simulations related to the anomalous thermal pixels indicated with an asterisk in the maps of figure 6. Figure 7 reports the comparison between the experimental curve and the 1D and 2D theoretical curves corresponding to the anomaly of figure 6a.The curves were obtained for a three-layer model made of mortar $(2 \mathrm{~cm})$, tuff $(3 \mathrm{~cm})$ and disrupted tuff $(2 \mathrm{~cm})$ whose thermal properties are indicated in table 3 . A good agreement is observed for both models, also if the match with the 1D curve exhibits a lower error than that observed for the 2D case. This result may be attributed to the position of the modeled pixel. Indeed, the pixel is located at the centre of a wide anomaly area, whose temperature variations along the selected x-axis may have not been captured by the 2D modeling. However, the modeling of the temperature transient allowed for defining both the nature and the depth of the thermal anomaly source, attributed to degradation of the tuff stone down to a depth of $7 \mathrm{~cm}$ below the structure surface.

Table 3. Material physical parameters used for the numerical simulations

\begin{tabular}{|c|c|c|c|c|}
\hline & $\mathbf{k}[\mathbf{W} /(\mathbf{m} \cdot \mathbf{K})]$ & $\mathbf{\rho}\left[\mathbf{k g} / \mathbf{m}^{\mathbf{3}}\right]$ & $\mathbf{C}[\mathbf{J} /(\mathbf{k g} \cdot \mathbf{K})]$ & $\mathbf{e}_{\mathbf{f}}\left[\mathbf{J} / \mathbf{m}^{\mathbf{2}} \mathbf{K \mathbf { s } ^ { \mathbf { 1 } }}\right]$ \\
\hline Mortar & 0.7 & 1600 & 840 & 969.95 \\
\hline Tuff & 1.7 & 2270 & 1000 & 1964.43 \\
\hline Disrupted Tuff & 1.5 & 2000 & 1300 & 1974.84 \\
\hline Sarno limestone & 0.7 & 1500 & 900 & 972.11 \\
\hline Lava & 3.3 & 2480 & 780 & 2526.56 \\
\hline Plaster & 1.1 & 1900 & 837 & 1322.62 \\
\hline Wet plaster & 0.7 & 2000 & 900 & 1122.50 \\
\hline marble & 2.3 & 2750 & 800 & 2249.44 \\
\hline
\end{tabular}




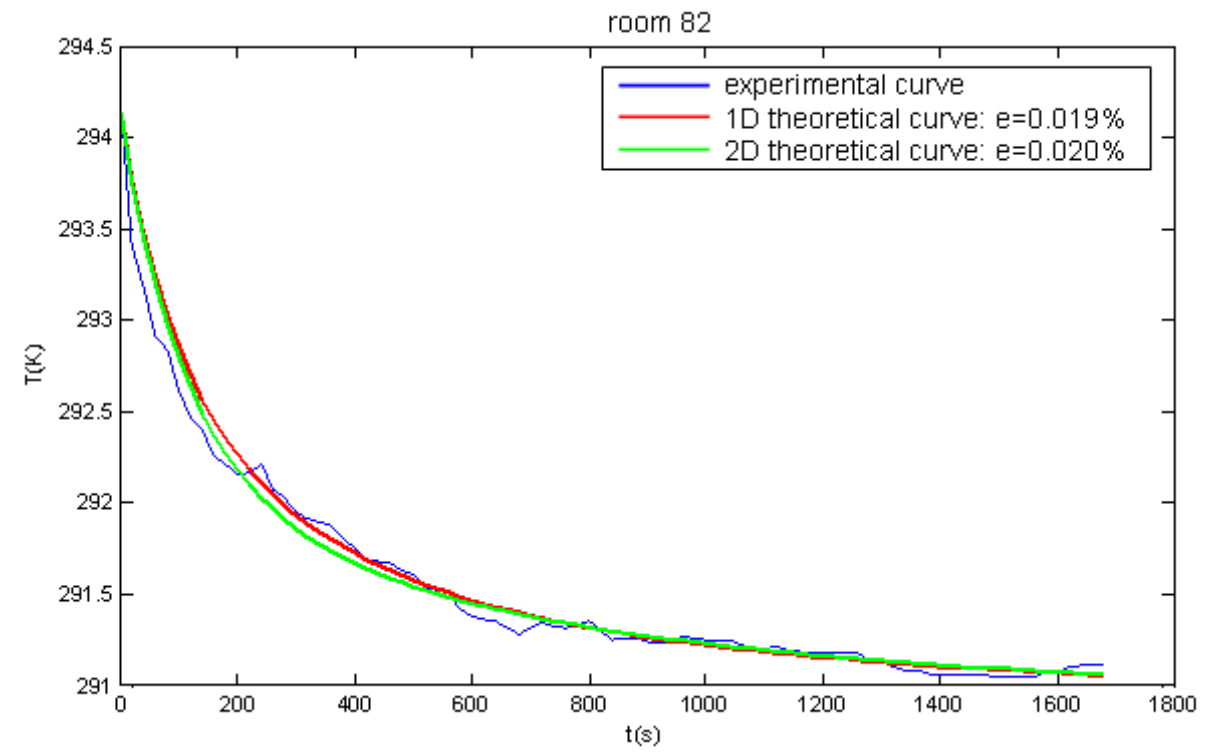

Fig. 7. Comparison between the experimental and theoretical temperature curves corresponding to the pixel indicated with an asterisk in figure 6a. The legend also indicates the percentage error (e)

Figure 8 shows the comparison between experimental and theoretical temperature decay curves relative to the pixel indicated with an asterisk in figure $6 \mathrm{~b}$. In this case the best result was obtained for a 2D model corresponding to a two-layer structure of plaster $(4 \mathrm{~cm})$ and marble $(3 \mathrm{~cm})$ (table 3$)$, and considering the temperature variations along the $x-$ axis. The simulation allowed for verifying the presence of a marble doorpost beneath the superficial layer of plaster.

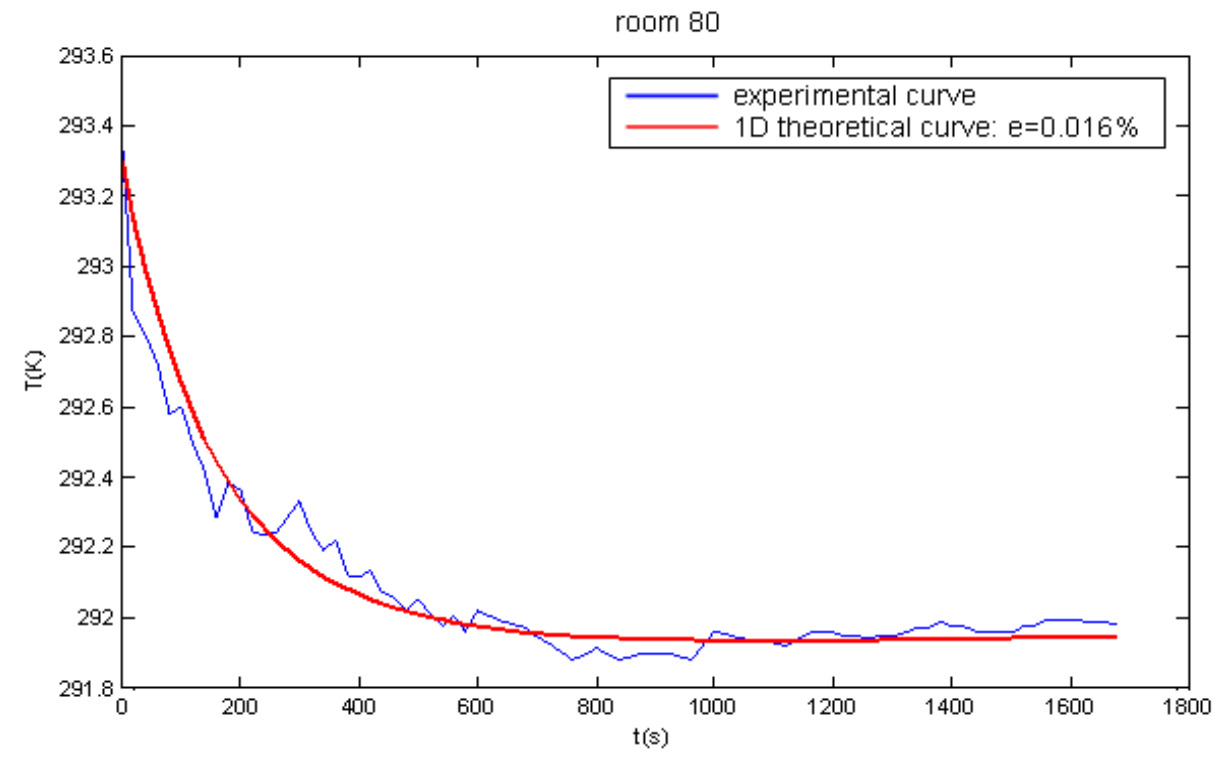

Fig. 8. Comparison between the experimental and theoretical temperature curves corresponding to the pixel indicated with an asterisk in figure $6 \mathrm{~b}$. The legend also indicates the percentage error (e)

\section{Conclusion}

Numerical codes for the forward thermal problem solution were developed. The application of the codes to the modeling of pulse-thermography laboratory data outlined their capability to distinguish with a high level of accuracy either the nature (density, thermal conductivity, specific heat) and/or the geometry (width, depth) of anomalies sources typical of architectonic structures.

The codes have been also used for quantitative interpretation of pulse-thermography data acquired on some walls of the Marcus Fabius Rufus' House (Pompeii, Italy). The main findings consist on the possibility to discriminate 
layered structures also in absence of evident thermal anomalies on the thermograms, and to identify the state of conservation of the constituent materials. The latter is very useful for future restoration works.

The disadvantage of the thermal data interpretation method here presented, as for all the iterative methods, is the use of a trial and error approach until a good match between experimental and theoretical curve is obtained. The development of a non-iterative inversion method is in fact actually in progress.

\section{REFERENCES}

[1] Maldague X.P.V., "Theory \& Practice of Infrared Technology for Nondestructive Testing". pp. 684, John WileyInterscience, New York, 2001.

[2] Meola C., Carlomagno G.M, "Recent advances in the use of infrared thermography". Measurement Science and Technology, vol. 15, pp. R27-R58, 2004.

[3] Meola C., "Infrared thermography of masonry structures". Infrared Physics and Technology, vol. 49, pp. 228233, 2007.

[4] Sun J.G., "Analysis of pulsed thermography methods for defect depth prediction". Journal of Heat Transfer, vol. 128, pp. 329-338, 2006.

[5] Delpech P.M., Krapez J.C., Balageas D.L., "Thermal defectometry using the temperature decay rate method". Proceedings of 2th Quantitative InfraRed Thermography conference, paper QIRT1994-033, Napoli (Italy), 1994.

[6] Krapez J.C., Maldague X., Cielo P., "Thermographic non-destructive evaluation: data inversion procedures: Part I - 1D analysis. Part II - 2D analysis and experimental results". Research Non-destructive Evaluation, vol. 3, pp. 81-100, 1991.

[7] Brink A., Maierhofer Ch., Rolling M.H., Weiggenhauser H., "Application of quantitative impulse thermography for structural evaluation in civil engineering - Comparison of experimental results and numerical simulations". Proceedings of 6th Quantitative InfraRed Thermography conference, paper QIRT2002-001, Dubrovnik (Croatia), D. Balageas, G. Busse, G.M. Carlomagno (Eds.), pp. 35-40, 2002.

[8] Maierhofer C., Brink A., Rollig H., Wiggenhauser H., "Quantitative Impulse-Thermography as non-destructive testing method in civil engineering - Experimental results and numerical simulations". Construction and Building Materials, vol. 19, pp. 731-737, 2005.

[9] Susa M., Ibarra-Castanedo C., Maldague X., Bendada A., Svaic S., Boras I., "Pulse thermography applied on a complex structure sample: comparison and analysis of numerical and experimental results". IV Conferencia Panamericana de END, Buenos Aires (Argentina), 2007.

[10] Lopez P., Sahli H., Vilarino D. L., Cabello D., "Detection of perturbations in thermal IR signatures: an inverse problem for buried land mine detection". Proceedings of EUDEM2-SCOT-2003: International Conference on Requirements and Technologies for the Detection, Removal, and Neutralization of Landmines and UXO, vol. 1, pp. 385-392, Brussels (Belgium), 2003.

[11] Thành N. T., Sahli H., Hào D. N., "Finite-difference methods and validity of a thermal model for landmine detection with soil property estimation". IEEE Trans. Geosci. Remote Sens., vol. 45, pp. 656-674, 2007.

[12] Weiser M., Röllig M., Arndt R., Erdmann B., "Development and test of a numerical model for pulse thermography in civil engineering". Heat and Mass Transfer, vol. 46, 2010.

[13] Heriansyah R., Abu-Bakar S.A.R., "Defect depth estimation in Passive Thermography using neural network paradigm". Proceeding of the WSEAS 6th International Conference on Circuits, Systems, Electronics, Control \& Signal Processing, pp. 421-425, Cairo (Egypt), 2007.

[14] Saintey M.B., Almond D.P., "An artificial neural network interpreter for transient thermography image data". NDT\&E International, vol. 5, pp. 291-295, 1997.

[15] D'Orazio T., Guaragnella C., Leo M., Spagnolo P., "Defect detection in aircraft composites by using a neural approach in the analysis of thermographic images". NDT\&E International, vol. 38, pp. 665-673, 2005.

[16] Recktenwald G. W., "Finite-Difference approximations to the Heat Equation". Mechanical Engineering, vol. 10, pp. 1-27, 2004.

[17] Gerya T.V., "Introduction to Numerical Geodynamic Modelling”. pp. 345, Cambridge University Press, New York, 2010.

[18] Grimaldi M., "VII 16 Insula Occidentalis 22. Casa di M. Fabius Rufus". In Pompei (Regiones VI-VII), Aoyagi M., Pappalardo U. editors, Insula Occidentalis, 257-418, Napoli, 2006.

[19] Di Maio R., Fedi M., La Manna M., Grimaldi M., Pappalardo U., "The contribution of the geophysical prospecting in the reconstruction of the buried ancient environments of the Marcus Fabius Rufus house (Pompeii, Italy)". Archaeological Prospection, vol. 17(4), pp. 259-269, 2010.

[20] Di Maio R., Meola C., Grimaldi M., Pappalardo U., "New insights for conservation of Villa Imperiale (Pompeii, Italy) through non-destructive exploration". International Journal of Architectural Heritage, 2012, in press. 